\title{
A brief overview of homologous recombination deficiency testing in cancers for the 'Next- Generation' Pathologist
}

\author{
Poornima Vijayan ${ }^{1}$, Luisa Bonilla ${ }^{1,2}$ \\ ${ }^{\prime}$ Department of Molecular Genetics, University of Toronto, Ontario, Canada \\ ${ }^{2}$ Department of Medical Oncology, Princess Margaret Cancer Centre, University Health Network, Toronto, Ontario, Canada
}

\author{
Keywords: \\ Cancer genomics; \\ Genomic scar \\ assay; Homologous \\ recombination deficiency; \\ Homologous \\ recombination repair \\ genes; \\ HRD status of tumors; \\ Mutational signatures; \\ PARPi responsive;
}

\begin{abstract}
Genomic instability is one of the hallmarks of cancer, having a crucial role in cancer pathogenesis as well as tumor proliferation. This essential feature is secondary to dysregulation of DNA damage repair pathways. Homologous repair represents the most reliable double-strand break repair mechanism. Homologous recombination deficiency is responsible for generating and perpetuating DNA damage in cancer, posing an opportunity for targeting treatment with poly(ADP-ribose) polymerase inhibitors through 'synthetic lethality', as well as platinum-based agents. Comprehensive genomic analysis has made it possible to discover molecular biomarkers that assist in the identification of Homologous recombination deficient tumors, allowing for the expansion of such treatment strategies to various other malignancies. Leveraging the improvement of genomic analysis methods to be more efficient in identifying Homologous recombination deficiency is crucial in the advancement of cancer care. The current review highlights the current strategies for Homologous recombination deficiency detection, clinical implications, limitations, and applicability.
\end{abstract}

\section{Correspondence:}

Dr. Poornima Vijayan

Department of Molecular Genetics, University of Toronto, Ontario, Canada ORCID ID: 0000-0001-8837-8718

Email: poornima.vijayan@mail.utoronto.ca

Received : July $3^{\text {rd }} 2020$; Accepted : August $16^{\text {th }} 2020$

Citation: Vijayan P, Bonilla L. A brief overview of homologous recombination deficiency testing in cancers for the 'Next-Generation' Pathologist. J Pathol Nep 2020;10: 1760-5. DOI: 10.3126/jpn. v10i2.29862

Copyright: This is an open-access article distributed under the terms of the Creative Commons Attribution 4.0 International License, which permits unrestricted use, distribution, and reproduction in any medium, provided the original author and source are credited.

\section{INTRODUCTION}

Cancer development is associated with oncogenic transformation led by genomic instability. ${ }^{1}$ Multiple genomic events are responsible for this transformation including mutations, copy number changes, and chromosomal changes, which can occur independently or conjunctively. Dysregulation of DNA damage repair (DDR) represents a fundamental feature of cancer pathogenesis and progression. ${ }^{1}$ This is in concordance with the estimation that two-thirds of cancer mutations occur due to DNA replication errors. ${ }^{2}$ DDR mechanisms include homologous recombination (HR) for the repair of double-strand breaks 


\begin{tabular}{ll|l|l|l|l|l}
\hline HRD \& Treatment Sensitivity & Genomic Alterations & HRD Assays & HTHWAY
\end{tabular}
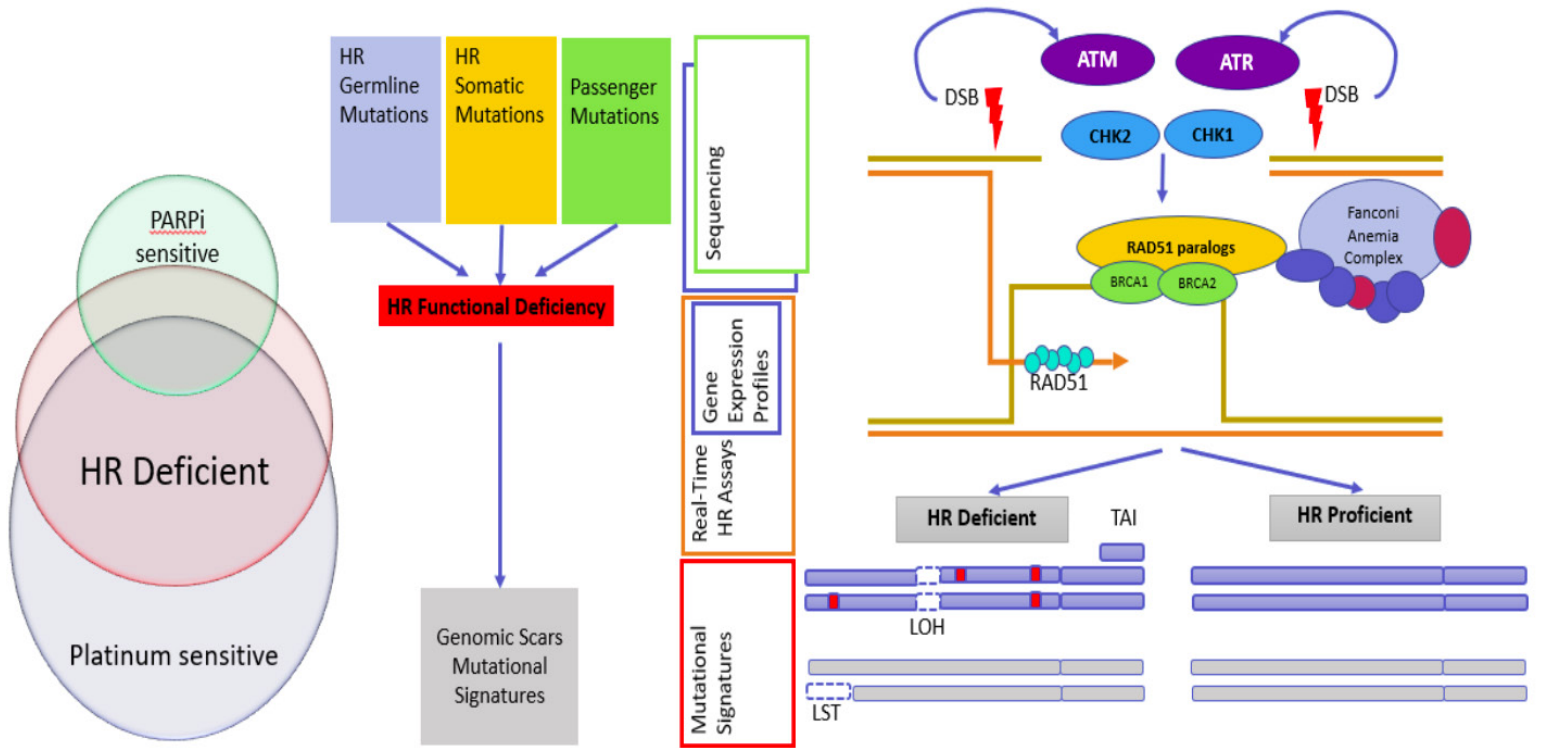

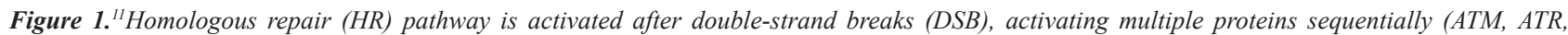
CHK1/2, RAD51, BRCA1/2, Fanconi anemic complex genes)to allow DNA high fidelity repair. Genomic alteration in the HR pathway leads to HR functional deficiency (HRD) causing loss of heterozygosity (LOH), large-scale transition (LST), telomeric allelic imbalance (TAI), establishing genomic scarring and unique mutational signatures. These are identifiable through multiple HRD assays, sequencing, gene expression profiles, real-time HR assays. Although there is considerable overlap between HRD status, platinum chemotherapy, and PARPi sensitivity, HRD is not an exclusive parameter for the prediction of response to these agents.

(DSB) among various other mechanisms..$^{3-5}$ Consequently, homologous recombination deficiencies (HRD) result in distinctive genomic alterations arising as a result of inherited and/or somatic mutations, that determine both genomic scarring and unique mutational signature patterns in various cancers. ${ }^{3-5}$ Recent advancements in genomic technology have helped capture these HRD related genomic scars and mutational-signatures to a certain extent and the determination of such 'tumoral HRD status' has been proven useful in driving treatment decisions. ${ }^{3-5}$

\section{TARGETED THERAPEUTIC APPLICATIONS OF HOMOLOGOUS RECOMBINATION DEFICIENCY IN CANCERS}

DNA repair pathways are indispensable to maintain DNA integrity and genomic stability in healthy tissues, and for resolving potentially lethal DNA damages. ${ }^{2}$ DNA DSBs are preferentially repaired by high fidelity HR pathway, while single-strand breaks (SSB) are repaired by the base excision repair (BER) pathway. ${ }^{6}$ PolyADP-ribose Polymerase 1 (PARP1) protein is critical for SSB repair via the BER pathway. ${ }^{6}$ BRCA1/2 genes encode for critical HR pathway proteins. ${ }^{6}$ Consequently, BRCA1/2 mutated tumors are HR deficient and are unable to repair damaged DNA. ${ }^{6}$ In this context, the event of PARP1 inhibition will lead to SSBs accumulation, eventually causing DSBs, which ultimately results in cell death due to the failure of the HR repair mechanism. ${ }^{6}$ This strategy of using PARP inhibitors to induce cell death in HR deficient tumors is called 'synthetic lethality'. ${ }^{6,7}$ Successful implementation of this therapeutic approach has been demonstrated in BRCA1/2 deficient breast, ovarian and prostatic cancers. ${ }^{7-9,11-15}$

Recent studies have demonstrated that in sporadic cancers, apart from BRCA1/2 mutations, dysregulation of any of the other critical proteins that make up the HR repair pathway such as RAD51, ATR, ATM, CHEK1, CHEK2, and Fanconi anemia complex genes (FANCD2, FANCA or FANCC 2) also causes HRD and confers such tumors with 'HRD status' or so-called 'BRCAness' phenotype (i.e, those tumors that may not have BRCA1/2 loss but show genomic alterations consistent with functional BRCA1/2 loss). ${ }^{6,8,11}$ Tumors with this BRCAness phenotype have been documented to benefit greatly from treatment with PARPi. ${ }^{6,8}$ Figure 1 shows a summary of the HRD mutational landscape including the various genes involved in the HR pathway, existing methodologies to detect the genomic alterations resulting from interruptions to the HR pathway, and the dynamics between HRD and treatment sensitivity to chemotherapeutic agents such as PARPi and platinum agents. ${ }^{11}$ Thus, it is clear that there exists a considerable overlap in the HRD targeting landscape and this can be leveraged to extend such treatment strategies to various other cancers that demonstrate HRD beyond familial breast/ ovarian cancers. 


\section{STRATEGIES TO DETECT HOMOLOGOUS RECOMBINATION DEFICIENCY}

Defects in DNA repair processes can lead to specific patterns of genomic alterations that can indicate a defect in a specific repair pathway or can lead to abnormal gene expression patterns. ${ }^{10,11}$ Many tumors harbor gross chromosomal rearrangements as a result of defective DNA repair and therefore assays have been developed to detect these so-called 'genomic scars', likely left behind by the loss of homologous recombination function. ${ }^{10,11}$ Different approaches are currently being investigated to identify HRD in tumors such as quantifying the extent of these genomic scars or by analysis of mutational signatures in specific tumors. ${ }^{10,11}$ Current strategies that are useful for detecting these genomic alterations employ high-throughput techniques for single nucleotide polypeptide (SNP) based genomic assays and molecular signature analysis. ${ }^{10,11}$

\section{SNP-BASED GENOMIC SCAR ASSAY}

Three types of SNP-based genomic assays are currently available to assess genomic scars in cancers, they include loss of heterozygosity $(\mathrm{LOH})$, telomeric allelic imbalances (TAI), and large-scale transitions (LST). ${ }^{11}$ Each of these quantifies a distinct type of genomic scar that is related to defective DNA repair. ${ }^{11}$ Thus, they can be considered as biomarkers for the state of DNA repair in particular cancer. Marquard et al analyzed the genomic scores of these biomarkers in 5371 tumor specimens across 15 cancer types and their study revealed a good correlation between the individual scores of the three biomarkers in different tumor types. Interestingly, cancers, where platinum therapy was the standard of care, showed significantly increased genomic scar scores. Additionally, they also found the presence of small subgroups of tumors with high genomic scar scores even in cancers where platinum was not the standard of care. ${ }^{18}$ Thus suggesting the potential therapeutic application of platinum chemotherapy in certain subgroups of patients with cancers that were not conventionally treated with platinum. ${ }^{18,19}$

Myriad Genetics offers 'myChoice HRD' test to detect genomic instability status in primary/metastatic breast and ovarian tumor samples. ${ }^{19}$ This test uses genomic DNA extracted from formalin-fixed paraffin-embedded (FFPE) tumor tissue. ${ }^{19}$ Genomic instability status assay is a hybridization capture panel that targets SNPs distributed across the whole genome. ${ }^{19}$ Next-generation sequencing (NGS) libraries are hybridized to this panel to enrich for sequences spanning these SNP locations and are sequenced using Illumina HiSeq next-generation sequencer. ${ }^{19}$ From the sequencing reads allele-specific copy number profiles are generated which are used for calculation of genomic instability score. ${ }^{19}$ This score takes into account $\mathrm{LOH}$, TAI, and LST. The results are presented as an 'HRD score' and tumors with scores above a validated cut-off $(>=42)$ are termed 'HRD positive'. ${ }^{19}$ This is useful in predicting response to neoadjuvant platinum chemotherapy in triple-negative breast cancer (TNBC). ${ }^{20}$ The NOVA study investigated the usefulness of the PARPi niraparib in platinum-sensitive ovarian cancer and found that patients with high HRD scores benefitted from it. ${ }^{21}$

\section{MUTATIONAL SIGNATURE ANALYSIS}

Somatic mutations can result from a plethora of exogenous (environmental) or endogenous (impaired DNA damage respond) events and each of these leave behind its unique imprint on the cancer genome called 'mutational signatures' ${ }^{19}$ Detecting and identifying mutational signatures have proven to be useful in guiding therapeutic decisions in various cancers. ${ }^{19}$ The availability of genome-wide mutational data across cancer genomes is very important for mutational signature analysis and NGS technologies have emerged as an important tool in this context. ${ }^{19}$ The capacity of NGS to analyze cancer genomes comprehensively has enabled the generation of comprehensive catalogs of somatic mutations in cancer patients. ${ }^{19}$ The most studied method of analysis of mutational signatures are based on base-pair substitutions and are characterized by the specific base change and its direct $5^{\prime}$ and $3^{\prime}$ flanking base. ${ }^{11,19}$ There can be 96 different combinations of trinucleotide changes given that there are six classes of base substitution and 16 possible sequence contexts. ${ }^{19}$ The mutational signatures can be deciphered from large volumes of NGS data from cancer patients by computational algorithms that recognize recurrent patterns using the '96-mutation matrix'. ${ }^{19}$ Thus, each pattern represents the relative proportion of each trinucleotide change, which translates to a mutational signature. ${ }^{19}$ In addition to base substitutions, other mutational events like indels, copy number variations (CNV), and structural rearrangements also have to be taken into account while analyzing the pattern of mutational signatures. ${ }^{19}$ Figure 2 provides a simplified overview of the utility of mutational signature analysis in the clinical setting. Figure adapted from Hoeck et al, 2019. ${ }^{19}$

Patient diagnosed with cancer undergoes biopsy from the tumor as well as from healthy tissue (e.g blood). Whole Genome Sequencing (WGS) technology is employed to sequence the DNA from both the samples for characterization of base substitutions, indels, structural rearrangements, and copy number variants (CNVs). The DNA from the healthy sample is used to characterize predisposition variants, while the cancer DNA sample provides for the characterization of medically actionable somatic driver mutational events. Thus a mutational signature pattern is identified, which either can directly support a specific cancer diagnosis in certain cases or influence clinical decisions such as referral for genetic counseling services, screening for family members, patientspecific treatment protocol selection, or prognostication. 

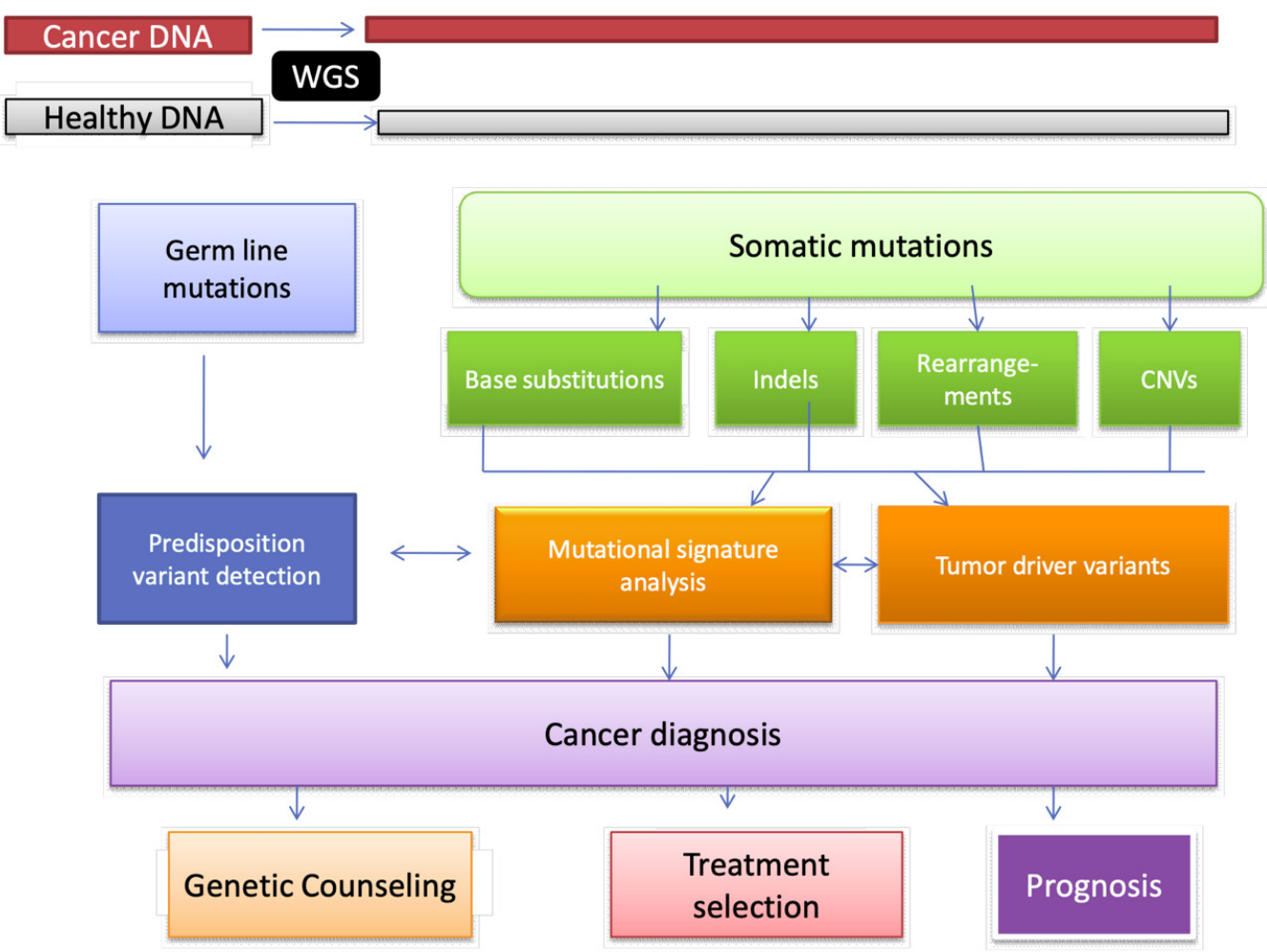

Figure $2^{19}$ : Overview of mutational signature analysis as a cancer diagnostic tool:

'Signature 3', first described by Alexander et al, is one such mutational signature that is attributable to HRD and is characterized by relatively equal proportions of all possible trinucleotide changes. ${ }^{3}$ It has been documented to exist in cancers involving many organs including breast, ovary, pancreas, and prostate. ${ }^{3}$ Currently, mutational-signature analysis based diagnosis is popular in the context of breast and ovarian cancers because of the well-documented evidence that BRCA1/2 loss in tumors correlates with HRD and thus renders them amenable to treatment with PARPi or DSB-inducing agents (platinum). ${ }^{19}$

Computational algorithms such as 'HRDetect' have been developed to accurately detect HRD in breast cancers based on their mutational signatures. ${ }^{21}$ HRDetect requires data from whole-genome sequencing. It needs inputs of the following information: mutational signature counts for each single base substitution, information on indels, and HRD score (sum of LOH, TAI, and LST scores). ${ }^{21}$ Using a machine learning algorithm (lasso regression), it assigns weighted values to these parameters and aggregates them into a single final score which predicts the degree of BRCA1/2 deficiency. ${ }^{21}$ In addition to identifying BRCA1/2 deficient tumors, HRDectect can identify tumors with 'BRCAness' with a sensitivity of almost $100 \% .{ }^{19}$ Thus, mutationalsignature analysis based tests such as HRDetect have the potential to find application in selecting patients who may benefit from treatment with PARPi or platinum agents in not just BRCA1/2 mutated breast/ovarian cancers but also various other cancer types associated with 'BRCAness'.

\section{ROLE CIRCULATING TUMOR DNA IN HOMOLOGUS RECOMBINATION DEFICIENCY DETECTION}

Targeted sequencing of circulating tumor DNA (ctDNA) is a minimally-invasive technique, wherein tumor cells circulating in the peripheral blood are isolated and analyzed for genomic aberrations. ${ }^{22}$ This strategy is now being increasingly used for early detection and monitoring progression in various cancers. Barbacioru et al, recently, developed a unique statistical method to identify the presence of $\mathrm{LOH}$ or somatic biallelic copy number loss of BRCA1/2 in ctDNA. ${ }^{23}$ The model was developed using coverage profiles and allelic frequencies of SNPs for each gene of interest and validated using in-silico tools. ${ }^{23}$ The model was applied to ctDNA samples of a large cohort of patients with advanced solid tumors and the results of this study were found to be highly concordant with the results from matched tissue samples. ${ }^{23}$ Another group demonstrated the utility of ctDNA in detecting clinically actionable genomic alterations in ovarian cancers ${ }^{22}$ A few clinical trials have used targeted sequencing of ctDNA for detecting HRD status in advanced prostatic cancers. ${ }^{6}$ Thus ctDNA-based strategies have great potential for clinical applicability in longitudinal monitoring of patients with advanced cancers given their minimally-invasive nature.

\section{CONCLUSIONS}

It is clear that HRD plays a crucial role in cancer 
pathogenesis and progression. ${ }^{4}$ Hence, accurate estimation of HRD status is essential, not only to guide treatment decisions but also for the development of novel therapeutic strategies, with the ultimate objective of expanding the pool of patients who may derive clinical benefit from such approaches. Although current approaches such as genomic scar assays and mutational signature analysis represent useful tools for determining tumoral HRD status, many of them are in their early stages of development requiring further validation studies. Therefore, there is an urgent need to further develop reliable HRD detection methodologies that are comprehensive, cost-effective, and minimallyinvasive with a high predictive value for treatment response and disease progression.

\section{Conflict of interest: None}

\section{REFERENCES}

1. Hanahan D, Weinberg RA. Hallmarks of Cancer: The Next Generation. Cell 2011;144: 646-74. Crossref

2. Curtin NJ. DNA repair dysregulation from cancer driver to therapeutic target. Nat. Rev. Cancer 2012;12:801-17. $\underline{\text { Crossref }}$

3. Alexandrov LB, Kim J, Haradhvala NJ, et al. The repertoire of mutational signatures in human cancer. Nature 2020;578:94-101. $\underline{\text { Crossref }}$

4. Ma J, Setton J, Lee NY, Riaz N, Powell SN. The therapeutic significance of mutational signatures from DNA repair deficiency in cancer. Nat. Commun. 2018;9:3292. $\underline{\text { Crossref }}$

5. Knijnenburg TA, Wang L, Zimmermann MT, et al. Genomic and Molecular Landscape of DNA Damage Repair Deficiency across The Cancer Genome Atlas. Cell Rep. 2018;23:239-54.e6. Crossref

6. Mateo J, Carreira S, Sandhu S, et al. DNA-repair defects and olaparib in metastatic prostate cancer. N Engl J Med 2015;373:1697-708. $\underline{\text { Crossref }}$

7. Pilié PG, Tang C, Mills GB, Yap TA. State-of-the-art strategies for targeting the DNA damage response in cancer. Nat. Rev. Clin. Oncol. 2019;16:81-104. $\underline{\text { Crossref }}$

8. Lord CJ, Ashworth A. BRCAness revisited. Nat. Rev. Cancer 2016;16:110-20. Crossref

9. Brown JS, O'Carrigan B, Jackson SP, Yap TA. Targeting DNA repair in cancer: Beyond PARP inhibitors. Cancer Discovery 2017;7:20-37. $\underline{\text { Crossref }}$
10. O'Kane GM, Connor AA, Gallinger S. Characterization, Detection, and Treatment Approaches for Homologous Recombination Deficiency in Cancer. Trends Mol. Med. 2017;23:1121-37. Crossref

11. Hoppe MM, Sundar R, Tan DSP, Jeyasekharan AD. Biomarkers for Homologous Recombination Deficiency in Cancer. JNCI J. Natl. Cancer Inst. 2018;110:704-13. Crossref

12. Forbes SA, Beare D, Boutselakis H, et al. COSMIC: somatic cancer genetics at high-resolution. Nucleic Acids Res.2016;45:D777-D783. Crossref

13. Evers B, Helleday T, Jonkers J. Targeting homologous recombination repair defects in cancer. Trends Pharmacol. Sci.2010;31:372-380. $\underline{\text { Crossref }}$

14. Srivas R, Shen JP, Yang CC, et al. A Network of Conserved Synthetic Lethal Interactions for Exploration of Precision Cancer Therapy. Mol. Cell 2016;63:514-25. $\underline{\text { Crossref }}$

15. Turner N, Tutt A, Ashworth A. Hallmarks of 'BRCAness' in sporadic cancers. Nat. Rev. Cancer 2004;4:814-19. $\underline{\text { Crossref }}$

16. Stefansson OA, Jonasson JG, Johannsson OT, et al. Genomic profiling of breast tumours in relation to BRCAabnormalities and phenotypes. Breast Cancer Research 2009;11. Crossref

17. Vollebergh M, Lips EH, Nederlof PM, et al. An aCGH classifier derived from BRCA1-mutated breast cancer and benefit of high-dose platinum-based chemotherapy in HER2-negative breast cancer patients. Annals of Oncology 2011;22:1561-70. $\underline{\text { Crossref }}$

18. Marquard AM, Eklund AC, Joshi T, et al. Pan-cancer analysis of genomic scar signatures associated with homologous recombination deficiency suggests novel indications for existing cancer drugs. Biomarker Research 2015;3. Crossref

19. Hoeck AV, Tjoonk NH, Boxtel RV, Cuppen E. Portrait of a cancer: mutational signature analyses for cancer diagnostics. BMC Cancer 2019;19. $\underline{\text { Crossref }}$

20. Telli ML, Timms KM, Reid J, et al. Homologous Recombination Deficiency (HRD) Score Predicts Response to Platinum-Containing Neoadjuvant Chemotherapy in Patients with Triple-Negative Breast Cancer. Clinical Cancer Research 2016;22:3764-73. $\underline{\text { Crossref }}$

21. Davies H, Glodzik D, Morganella S, et al. HRDetect is a predictor of BRCA1 and BRCA2 deficiency based on 
mutational signatures. Nature Medicine 2017;23:517-25. $\underline{\text { Crossref }}$

22. Oikkonen J, Zhang K, Salminen L, et al. Prospective Longitudinal ctDNA Workflow Reveals Clinically Actionable Alterations in Ovarian Cancer. JCO Precision Oncology 2009;1-12. Crossref
23. Barbacioru C, Raymond VM, Sikora M, et al. Abstract 435: Cell-free circulating tumor DNA (ctDNA) detects somatic copy number loss in homologous recombination repair genes. Clinical Research (Excluding Clinical Trials) 2019. $\underline{\text { Crossref }}$ 